\title{
The Aggressive Surgical Treatment and Outcome of a Colon Cancer Patient Infected with SARS-CoV-2 in Wuhan, China and Our Experience Sharing
}

\section{Jinbo Gao}

Huazhong University of Science and Technology

\section{Ming Yang}

Huazhong University of Science and Technology

Lian Liu

Huazhong University of Science and Technology

\section{Shuang Guo}

Huazhong University of Science and Technology

\section{Yongfeng Li}

Huazhong University of Science and Technology

Chao Cheng ( $\sim$ chengcha00306@sina.cn)

Huazhong University of Science and Technology

\section{Case Report}

Keywords: Colon Cancer, COVID-19, SARS-CoV-2, Pathology, Case report

Posted Date: April 16th, 2020

DOI: https://doi.org/10.21203/rs.3.rs-23214/v1

License: (c) (i) This work is licensed under a Creative Commons Attribution 4.0 International License.

Read Full License 


\section{Abstract}

Background: Cancer patients are at increased risks of novelcoronavirus disease 2019 (COVID-19). Currently, surgical strategies for cancer patients with COVID-19 are generally suggested to be properly delayed.

Case presentation:We presented a 69-year-old Chinese female colon cancer patientwith COVID-19, the first case accepted the surgical treatment during the epidemic season in China. Thepatient developed a fever on January 28, 2020. After treatments with Ceftriaxone and Abidol, her fever was not reduced yet. A repeat chest computed tomography (CT) scan showed the infectious lesions significantly exacerbated, with a positive result for severe acute respiratory syndrome coronavirus 2 (SARS-CoV-2) nucleic acid. An abdomen CT scan indicated the tumor of ascending colon with local wrapped changes. She was diagnosed with 'Severe novel coronavirus pneumonia' and 'Incomplete bowel obstruction: Colon cancer?'. After actively anti-inflammatory and anti-viral therapies, aright colectomy with lymph node dissection was performed on March 11. The pathological changes of tissue specimens were further investigated.The patient successfully recovered from COVID-19 and surgery, without any postoperative related complications, and was discharged on the 9th day after operation. No case of surgeon, nurse or anesthetist in our team infected by SARS-CoV-2 occurred. Microscopically, significant degeneration, necrosis and slough of focal intestinal and colonic mucosal epithelial cells were observed.

Conclusions:It is meaningful and imperative to share our experience to protect health care personnel from SARS-CoV-2infection and to provide references for optimizing treatment of cancer patient, at least for the operative intervention absolutely necessary or emergency surgery,during the outbreak of COVID19.

\section{Background}

The rapid spread of the novel coronavirus 2019-nCov, designated as severe acute respiratory syndrome coronavirus 2 (SARS-CoV-2) by the Coronavirus Study Group of the International Committee on Taxonomy of Viruses on February 12, 2020, has resulted in an ongoing outbreak of viral pneumonia and rapidly spreading across the world since December 2019 , which has been labeled as a public health emergency of international concern by the World Health Organization [1, 2]. Patients suffered from severe or critical novel coronavirus pneumonia showed a higher mortality rate, associating with older age and underlying comorbidities (such as diabetes, cardiovascular and cerebrovascular diseases) [3]. Notably, due to the systemic immunosuppressive status caused by the malignancy, patients with cancer are at increased risks of severe infections and have a poorer prognosis [4]. Currently, several conservative treatment strategies have been recommended for patients with colorectal cancer under the background of coronavirus pneumonia, whereas surgery for patients with surgical indications are generally suggested to be properly delayed [5]. On the other hand, concerning deteriorating infection, most patients with cancer were suggested to withdraw cancer treatment after SARS-CoV-2 infection, such as chemotherapy, radiotherapy and targeted therapy [6]. Therefore, patients with cancer suffered from a relatively increased 
risk of tumor progression, making the issue of treatment options for tumor cases controversial. Here, we firstly reported a colon cancer case infected with SARS-CoV-2 underwent radical resection of right colon without postoperative related complications and recovered from novel coronavirus pneumonia, detailed pathological findings and shared our experience during the perioperative period.

\section{Case Presentation}

A Chinese female patient, aged 69 years, presented with intractable intermittent fever, dry cough, chest tightness and right lower abdomen pain for two days when was referred to our hospital for further novel coronavirus pneumonia and cancer treatments. On January 28, 2020, a fever developed in the patient $\left(38.4^{\circ} \mathrm{C}\right)$, accompanied by fatigue and muscle soreness. This patient denied any medical, trauma or surgical history. Laboratory tests indicated slightly increased neutrophil percentage $(74.0 \%)$ and decreased lymphocyte percentage (18.6\%). A chest computed tomography (CT) scan on February 1 showed a few ground-glass opacities in the lower lobes of both lungs, suggesting suspect for 'infectious lesions of both lungs' (Fig.1A). Tests for influenza virus and other infectious agents were negative. However, the novel coronavirus nucleic acid test was not taken due to her mild symptoms and lack of nucleic acid detection kits. After treatment with Ceftriaxone and Abidol, her fever was not reduced yet. On February 8 , a repeat CT scan showed ground-glass opacity lesions significantly increased as compared with the previous one (Fig.1A). Laboratory tests revealed remarkably decreased lymphocyte count (0.63 $\mathrm{G} / \mathrm{L})$, but normal neutrophil count $(4.38 \mathrm{G} / \mathrm{L})$. With adding methylprednisolone to the original treatment, patient's fever gradually reduced to normal. Although the patient denied any contact with confirmed case in this period, a throat swab was obtained and the nucleic acid test for SARS-CoV-2 came back positively On February 20. However, she reappeared with fever on February 24, with a body temperature of up to $38.3^{\circ} \mathrm{C}$, and accompanied by dry cough, chest tightness and abdominal pain (especially in the right lower abdomen). Due to poor treatment effect in the local hospital, she was referred to the infectious disease ward in our hospital on February 26. An abdomen CT scan indicated the tumorous lesion of the ascending colon with perforated and local wrapped changes on February 27 (Fig.1B). The patient was diagnosed with 'Severe novel coronavirus pneumonia' and 'Incomplete bowel obstruction: Colon cancer?'. Thereafter, the patient started treatment for novel coronavirus pneumonia with Abidol and Moxifloxacin, as well as the corresponding symptomatic and nutritional support therapies. After above treatments, however, the patient still presented with intermittent fever, whereas other accompanying symptoms were significantly relieved since February 29. Paradoxically, the follow-up results of nucleic acid retest for SARS-CoV-2 were negative on February 29, March 2 and March 10, respectively, with positive results of serum SARS-CoV-2 IgG antibodies on March 2, suggesting recovery from novel coronavirus pneumonia.

Considering the fever probably caused by tumor, the risk of tumor progression and likely recurrences of abdominal pain, and even developing to complete bowel obstruction, which could make the general condition worse or be readmitted again, an aggressive surgical treatment was recommend for the patient. Furthermore, the patient did however present extreme motivation for surgery in an excellent general condition, optimistic mental state and well-nourished despite the weight loss throughout the whole treatment period. Additionally, no other comorbidity factors contraindicated the operation. Based on 
carefully preoperative evaluation and preparation, our team performed an exploratory laparotomy for this patient via continuous epidural anesthesia, which took about 3.5 hours, on March 11, 2020. Upon entering the abdominal cavity through a midline incision, we found that the tumor was approximately $6.0 \mathrm{~cm} \times 5.0 \mathrm{~cm}$, originating from the starting portion of ascending colon. The lesion was wrapped by omentum and surrounding tissues and accompanied by inflammatory adhesions to lateral abdominal wall and a portion of terminal ileum, which was consistent with CT scan results (Fig.1B). No invasion to surrounding organs was found. Therefore, the right colectomy with lymph node dissection was performed. Five days after surgery, she could exhaust and defecate from the anus, suggesting relatively late recovery of bowel function. As of March 20 , she gradually recovered without postoperative complications and was discharged on the 9th day after operation. Currently, the patient is being followed up closely as an outpatient.

The pathologic examination found a $5.0 \mathrm{~cm} \times 5.0 \mathrm{~cm}$ ulcerative mass in the ileocecal area $1 \mathrm{~cm}$ from ileocecal valve, showing a gray white surface and necrosis in the center grossly. Microscopically, tumor invaded into the subserosal adipose tissue without lymph node metastasis, diagnosed as mucinous adenocarcinoma in the histologic diagnosis with pT3NO (Fig.1C). Of note, the significant degeneration, necrosis and slough of focal intestinal and colonic mucosal epithelial cells were observed in terminal ileum and ileocecal area surrounding the tumor, respectively (Fig.1C).

\section{Discussion And Conclusions}

The rapidly ongoing outbreak of novel coronavirus disease 2019 (COVID-19), caused by SARS-CoV-2, has attracted widespread concern not only within China but around the globe [1, 2]. As for cancer patients with COVID-19, whether it should discontinue cancer therapy or defer surgical treatment remains controversial. To our knowledge, the case reported here represented the first colon cancer patient with confirmed COVID-19, who successfully underwent and benefited from the aggressive surgical treatment. During the period of manuscript preparation, no more surgery was performed for colon cancer patient infected with SARS-CoV-2, and thus clinic data on safety of surgical treatment and prognosis of such patients are sorely lacking. However, there are still positive implications and certain guiding significances for the treatment of colon cancer cases with emergency situation during the health care crisis.

Although the radical right colectomy is a standard surgical procedure for gastrointestinal surgeons, it remains physically and technically challenging that performing the operation for colon cancer patient with confirmed SARS-CoV-2 infection in the specific period, because of its complexity which includes prolonged operative time and unclear vision, as well as discomfort and inconvenience caused by multiple sets of protective equipment. More importantly, several reasons make invasive diagnostic and surgical procedures less of a clinical priority, such as suddenness of the outbreak, high rate of transmission, vast patient volume in hospitals, and severe shortage of health care personnel [3], which indeed increases infection risk and mental stress for the medical team. In this case report, we successfully performed the operation for this patient with confirmed COVID-19. Everyone in our surgical team ensured to implement the standard tertiary protective measures against infectious diseases and applied active iodine on the 
surface of medical goggles to ensure a good surgical field, and then checked again with each other. Furthermore, we especially focused on responding to critical situations such as major bleeding, respiratory and cardiac arrest during the operation. Another two surgeons were on call at all times during surgery to deal with the shortage of surgical member due to significantly prolonged surgery time and excessive physical exertion of the surgeon. In order to minimize the production of iatrogenic aerosols, the anesthesiologist used continuous epidural anesthesia, but meanwhile have prepared the equipment required for general anesthesia and rescue for timely responding to emergencies such as anesthesia accidents and respiratory distress during the surgery. Moreover, surgery must be performed in a negative pressure operating room. Before entering the abdominal cavity, the operation must be gentle to prevent body fluid from splashing, which was also applicable during tissue dissection and surgical specimen removal.

A previous study has demonstrated that combining antiviral and anti-inflammatory treatments could simultaneously reduce viral infectivity, viral replication, and the aberrant host inflammatory response [7]. Therefore, we recommended the preoperative treatment with combination of antiviral and antiinflammatory medicine, so that patients could benefit great in the processes of undergoing surgery and postoperative rehabilitation. Of note, there are some special patients that has been cured and discharged recently appeared in Wuhan, China, whose retesting results of nucleic acid for SARS-CoV-2 were positive again during outpatient follow-up and was re-admitted to the hospital. Therefore, it is prudent and important to regard all patients as potentially infectious during an epidemic season and cautiously practice "universal precaution" in perioperative period. Fortunately, thus far, no case of surgeon, nurse or anesthetist in our team being infected by SARS-CoV-2 occurred.

During the earlier phase of the COVID-19 outbreak, there were a significant number of health care providers infected with SARS-CoV-2 in many hospitals in Wuhan, and patients in a same room were cross-infected, due to exposure to unknown transmission sources. This dramatically increased the risk of SARS-CoV-2 infection for everyone in the outpatient clinics, especially cancer patients. On retrospective analysis of the patient's medical course, the underlying cause of the first fever might be tumor-derived, and then were cross-infected with SARS-CoV-2 due to outpatient exposure. The SARS-CoV-2 infection and tumor lesion together caused a synergistic mechanism of action and further aggravated the patient's condition, which partly was clarified by the fever eventually reduced to normal after surgery. Pathologic findings revealed that the obvious degeneration, necrosis and slough of focal intestinal and colonic mucosal epithelial cells surrounding the tumor, which was consistent with the findings in a previous autopsy report [8]. Based on many cases with diarrhea observed clinically, it is probably one of evidences for gastrointestinal infection of SARS-CoV-2. However, these changes might also represent a nonspecific change with aging and could be considered as a largely secondary change caused by tumor. More cases with sufficient controls are necessary to further clarify these pathological changes. Nevertheless, it is of great significance for clinicians to pay more attention to cancer patients and pathological changes of digestive tract organs during the outbreak of COVID-19. We believe that it is meaningful and imperative to share our experience to provide references for optimizing treatment of cancer, at least for operative intervention which is absolutely necessary during the epidemic period. 


\section{Abbreviations}

COVID-19, novel coronavirus disease 2019;

CT, computed tomography;

SARS-CoV-2, severe acute respiratory syndrome coronavirus 2

\section{Declarations}

\section{Ethics approval and consent to participate}

The study was approved by the ethics institutional review board of the Union Hospital of Huazhong University of Science and Technology. Informed consent was obtained from the participant included in the study.

\section{Consent for publication}

The patient has signed informed consent regarding publishing her data and photographs.

\section{Availability of data and materials}

The data and materials used to support the findings of this study are included within the article.

\section{Competing interests}

The authors declare that they have no competing interests.

\section{Funding}

This study was partially supported by research funding from the Fundamental Research Funds for the Central Universities (Grant No. 2016YXMS250) and Natural Science Foundation of Hubei Province (Grant No. 2016CFB228).

\section{Author contributions}

Performed surgery: JG, LL, YL and CC; Pathological examination: MY and SG;

Design of the work: JG; Data collection: LL; Postoperative management: YL

Writing - original draft preparation: CC; Writing - review and editing: JG

\section{Acknowledgements}

Not applicable. 


\section{References}

1. Wang C, Horby PW, Hayden FG, Gao GF: A novel coronavirus outbreak of global health concern. Lancet 2020, 395(10223):470-473.

2. Perlman S: Another Decade, Another Coronavirus. N Engl J Med 2020, 382(8):760-762.

3. Tian S, Hu W, Niu L, Liu H, Xu H, Xiao SY: Pulmonary pathology of early phase 2019 novel coronavirus (COVID-19) pneumonia in two patients with lung cancer. J Thorac Oncol 2020.

4. Liang W, Guan W, Chen R, Wang W, Li J, Xu K, Li C, Ai Q, Lu W, Liang H et al: Cancer patients in SARSCoV-2 infection: a nationwide analysis in China. Lancet Oncol 2020, 21(3):335-337.

5. Yu GY, Lou Z, Zhang W: [Several suggestion of operation for colorectal cancer under the outbreak of Corona Virus Disease 19 in China]. Zhonghua Wei Chang Wai Ke Za Zhi 2020, 23(3):9-11.

6. Zhang H, Huang Y, Xie C: The Treatment and Outcome of a Lung Cancer Patient Infected with SARSCoV-2. J Thorac Oncol 2020.

7. Stebbing J, Phelan A, Griffin I, Tucker C, Oechsle O, Smith D, Richardson P: COVID-19: combining antiviral and anti-inflammatory treatments. Lancet Infect Dis 2020.

8. Yao XH, Li TY, He ZC, Ping YF, Liu HW, Yu SC, Mou HM, Wang LH, Zhang HR, Fu WJ et al: [A pathological report of three COVID-19 cases by minimally invasive autopsies]. Zhonghua Bing Li Xue Za Zhi 2020, 49(0):E009.

\section{Figures}




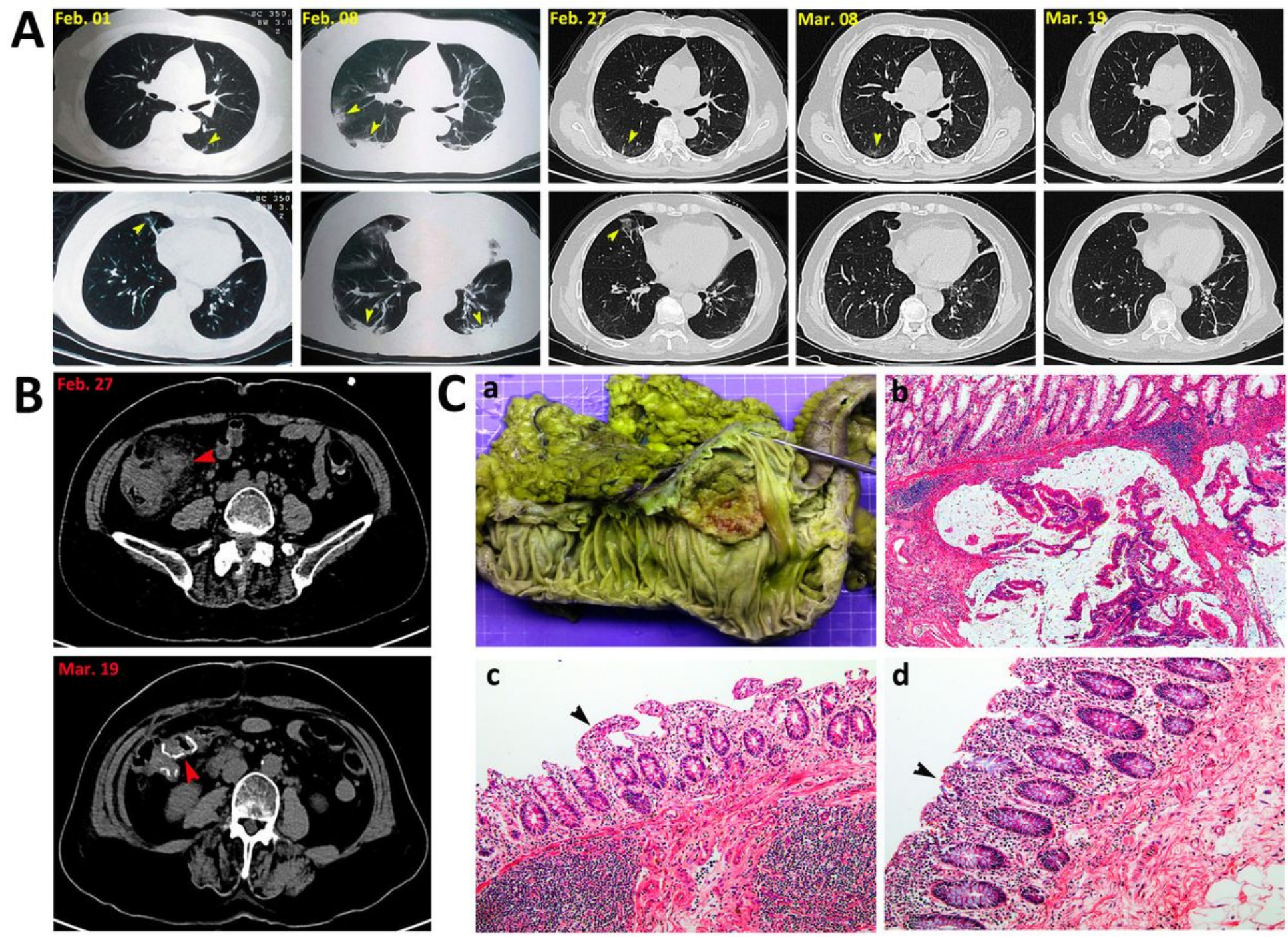

Figure 1

Representative images of chest CT scans and pathologic assessment. (A) Chest CT scans during the patient's clinical course. The yellow arrows indicated patchy shadows in both lungs. (B) Preoperative CT scans on February 27 showed tumor extension, and postoperative CT scans indicated well-healed anastomosis on March 11, 2020. The red arrows indicate tumors or anastomosis, respectively. (C) Pathologic assessment of tissue specimen from colon cancer patient with SARS-CoV-2 infection. (a) The cut surface showed an ulcerative mass and necrosis in the center. (b) The histologic diagnosis of mucinous adenocarcinoma (H\&E; 40x). (c) Degeneration, necrosis and slough of focal mucosal epithelial cells in terminal ileum, and (d) in colon of the ileocecal area (H\&E;100x). The black arrows indicated histologic changes. CT = computed tomography; H\&E = Hematoxylin \& eosin staining 